\title{
BODY CHANGES IN WOMEN UNDERGOING CHEMOTHERAPY FOR BREAST CANCER
}

\author{
by \\ Kristin Leigh Campbell \\ B.Sc.PT (Hons), Queen's University, 1997 \\ A thesis submitted in partial fulfillment of the requirements for the \\ degree of \\ Master of Science \\ in \\ The Faculty of Gradate Studies \\ School of Human Kinetics
}

We accept this thesis as conforming to the required standard

The University of British Columbia

November 2001

(C) Kristin Leigh Campbell, 2001 
In presenting this thesis in partial fulfilment of the requirements for an advanced degree at the University of British Columbia, I agree that the Library shall make it freely available for reference and study. I further agree that permission for extensive copying of this thesis for scholarly purposes may be granted by the head of my department or by his or her representatives. It is understood that copying or publication of this thesis for financial gain shall not be allowed without my written permission.

Department of HUMAN KINETICS:

The University of British Columbia Vancouver, Canada

Date $\operatorname{Nov} \cdot 16,2001$ 


\section{Abstract}

Contrary to the general assumptions about the effects of cancer treatment, women undergoing adjuvant chemotherapy treatment for breast cancer tend to gain weight rather than lose it. This weight gain has implications for other health outcomes, disease-free survival, and psychosocial sequelae. Changes in body composition, resting metabolic rate, physical activity, dietary intake and menopausal status have all been associated with this weight gain. The purpose of this study was to better understand the mechanism of the weight gain by looking at the impact of possible body composition changes and changes in resting metabolic rate that occur with adjuvant chemotherapy treatment. Methods: Weight, resting metabolic rate, and menopausal status were measured prior to treatment, and with each cycle of chemotherapy in a group of five women (mean age $49.2 \pm 5.4$ years; mean weight $75.2 \pm 17.8 \mathrm{~kg}$ ) undergoing adjuvant chemotherapy for stage I-IIIA breast cancer. Body composition was measured prior to the start of chemotherapy and after the last cycle, using duel-energy $x$-ray absorptiometry. Results: One-way repeated measures ANOVA did not show any significant difference in weight or resting metabolic rate throughout treatment, however, plots of the mean effect showed an increase in weight over time and a decrease in resting metabolic rate during treatment, which returned to pre-treatment levels post treatment. However, the combined slopes of individual regression lines did not differ from zero. Average weight gain was $1.9 \mathrm{~kg}$ (range -2.71 to 6.81 ). No significant changes in body composition were found, however, a $2.5 \%$ increase 
in total percent body fat, and $3.5 \%$ increase in trunk percent body fat approached significance, along with a loss of left arm lean mass and gain in left leg lean mass. All participants became amenorrheic during treatment, except one that was postmenopausal prior to treatment. Conclusion: Participants tended to gain weight with treatment. The most striking association was the development of chemotherapy induced amenorrhea, which has been linked to changes associated with natural menopause, namely weight gain, a loss of lean mass, gain of fat mass, and change in fat distribution. 


\section{Table of Contents}

Abstract ii

Table of Contents $\quad$ iv

List of Tables vi

List of Figures vii

Acknowledgements viii

Chapter 1: Introduction 1

$\begin{array}{ll}\text { Research Hypothesis } & 1\end{array}$

Chapter 2: Methodology 3

$\begin{array}{ll}\text { Subjects } & 3\end{array}$

$\begin{array}{ll}\text { Measurements } & 4\end{array}$

$\begin{array}{ll}\text { Logistics } & 6\end{array}$

$\begin{array}{ll}\text { Statistical Analysis } & 8\end{array}$

Chapter 3: Results 9

$\begin{array}{lr}\text { Testing } & 10\end{array}$

$\begin{array}{ll}\text { Weight Change } & 11\end{array}$

$\begin{array}{lr}\text { Resting Metabolic Rate } & 13\end{array}$

$\begin{array}{ll}\text { Body Composition } & 16\end{array}$

$\begin{array}{ll}\text { Menstrual Status } & 17\end{array}$

$\begin{array}{ll}\text { Chapter 4: Discussion } & 18\end{array}$

Summary of Individual Results $\quad 24$

$\begin{array}{ll}\text { Summary } & 25\end{array}$

$\begin{array}{ll}\text { Limitations } & 25\end{array}$ 
Future Recommendations

References

Appendix A: Literature Review

I. Breast cancer demographics and current treatment

II. Sequelae of weight gain

III. Postulated mechanisms of weight gain

A. Energy Balance

i. Resting Metabolic Rate

ii. Thermogenesis

iii. Physical Activity

iv. Dietary Intake

B. Hormonal Factors

i. Menopausal Status

40

ii. Hormonal Milieu

41

IV. Previous Investigations

Research Limitations

Summary

Appendix B: TNM Staging for breast cancer 52

Appendix C: DXA Reliability study 54

Methods $\quad 54$

Results $\quad 55$

$\begin{array}{ll}\text { Conclusion } & 57\end{array}$

Appendix D: Raw Data $\quad 59$ 


\section{List of Tables}

Table 1 Surgical results with pathology and staging results of cancer 10

$\begin{array}{lll}\text { Table } 2 \text { Chemotherapy regimes } & 10\end{array}$

Table 3 Weight before and at the end of treatment $(\mathrm{kg})$, plus change 13

Table 4 Mean resting metabolic rate across treatment time in $\mathrm{kcal} / \mathrm{d}$ (standard deviation)

Table 5 Measures of total fat mass, lean mass, and percent body fat before and after treatment and for those areas approaching significance, using DXA (g)

Table 6 Presence of a menstrual period throughout treatment

Table 7 Percent coefficient of variation (\%CV) and standard deviation (SD) for fat mass, lean mass, total mass, and percent body fat.

Table $8 \quad$ Participant characteristics

Table 9 Weight change over the course of treatment $(\mathrm{kg})$

Table 10 Change in resting metabolic rate over the course of Treatment (kcal/day) 


\section{List of Figures}

$\begin{array}{lll}\text { Figure 1a Weight Main Effect } & 12\end{array}$

Figure 1b Weight Main Effect (CEF only) 12

$\begin{array}{lll}\text { Figure 2a Resting Metabolic Rate Main Effect } & 14\end{array}$

Figure 2b Resting Metabolic Rate Main Effect (CEF only) 14

Figure 3 Resting Metabolic Rate for all participants over treatment time 


\section{Acknowledgements}

Dr. Don McKenzie My advisor, who always said everything would work out eventually and it always did.

My Committee Dr. Alan Martin and Dr. Karen Gelmon whose contributions made this project possible.

The Participants Who welcomed me into their homes, at far too early an hour in the morning, and while they were undergoing treatment.

BCCA Staff

Dr. Chia, Dr. Shenkier, and all the residents, nursing practioners and support staff who helped to recruit study participants. Without them, there would have been no study.

Diana Jesperson

Who was always there to answer questions and figure out where things had gone wrong.

Cheri Van Patten The voice of experience who always made the time to pass her knowledge on to me.

My parents

Who always encouraged me (weight is mass, isn't?)

Susan Harris

One of the main reasons I ever thought of doing a Masters in the first place, and a constant help throughout the process.

Ian Stewart

How he put up with all my questions l'll never know

Darren Warburton Who also answered more than his fair share of questions and made time to help me along my way

Jane LaBreche It is nice to have someone traveling along the same road at the same time, especially one with such a sense of humour.

Andrew Attwell A valued colleague and friend, who always encourage me and listened to my research ideas.

Akosua Tomash Who went out of her way to help.

Greg Scutt Who knew I could do it, just wished I was better at math 


\section{Chapter 1 - INTRODUCTION}

Contrary to the general assumptions about the effects of cancer treatment, women undergoing adjuvant chemotherapy treatment for breast cancer tend to gain weight, rather than lose it. Significant weight gain has been noted to occur in $50-96 \%$ of all patients with early-stage breast cancer receiving adjuvant chemotherapy 1 . Weight gain of $2.5 \mathrm{~kg}$ to $6.2 \mathrm{~kg}$ appears to be most common, with gains of more than $10 \mathrm{~kg}$ not being unusual1, 2 . Body composition as measured by percent of body fat and lean body mass, also appears to be altered, with a tendency towards an increase in fat mass and a decrease in lean body mass $3-5$.

The observed weight gain has been shown to have a significant impact on the lives of women, specifically in terms of other health outcomes, diseasefree survival, and psychosocial sequelae. Therefore, weight gain is a significant and potentially serious problem for women undergoing this type of cancer treatment. However, the mechanisms involved in this weight gain are not well understood, making effective intervention difficult.

Research Hypothesis:

The purpose of this study was to investigate the mechanisms responsible for weight gain in women undergoing chemotherapy for stage I-IIIA breast cancer treatment. From the literature, changes in several factors have been linked to weight gain, namely body composition, hormonal milieu, menstrual status, resting metabolic rate, energy intake, and energy expenditure. We chose 
to focus on weight, resting metabolic rate, body composition, and menstrual status. The hypothesis of this investigation was that women would gain weight over the course of adjuvant chemotherapy treatment for breast cancer. As well, of those who were premenopausal prior to treatment, the majority would develop amenorrhea with treatment. A gain in fat mass and loss of lean body mass was also expected, along with a possible decrease in resting metabolic rate during treatment. 


\section{Chapter 2 - METHODOLOGY}

This research project was a descriptive correlational study design, which looked at some of the factors associated with weight gain in women undergoing adjuvant chemotherapy for breast cancer treatment. All measurements were performed pretreatment and post treatment, with weight and resting metabolic rate also measured once per cycle of chemotherapy (every 3-4 weeks). $\underline{\text { Subjects }}$

The population under study was women with early stage (I-IIIA) operable breast cancer, which was followed by an adjuvant chemotherapy regime under the supervision of an attending oncologist at the Vancouver Cancer Centre (VCC) of the British Columbia Cancer Agency (BCCA). All gave signed informed consent once the methodology of the study was explained. Using values from the literature, a power calculation based on body weight was performed to estimate the probability of finding a significant effect if there was a true difference over time. The average weight gain has been noted as $4.35 \mathrm{~kg}$, with a standard deviation of $5.65 \mathrm{~kg}$. Using a one-tailed test, with alpha level .05 , a sample size of 20 , with repeated measures, will give a power of $80 \%$. This sample size was not reached during the first phase of recruitment. The reality of cancer diagnosis and treatment, along with concurrent recruitment for numerous other trials, and health care sector labour disputes, contributed significantly to the low number of participants included in this analysis. 
Inclusion criteria for the population under study were as follows: 18 to 70 years of age at the time of study, ability to communicate in English, accessible geographically, in good general health, non-smoker, and with a stable weight prior to entry into the study. These criteria were chosen to ensure that the subjects would be of sufficient age to give informed consent, would be able to adequately complete the initial questionnaire, and have few factors, beyond those under study, that may influence body weight.

Exclusion criteria were: receiving estrogen replacement therapy or tamoxifen, long-term use of medications that promote weight gain, or with serious co-morbid medical illnesses. The use of estrogen replacement therapy or tamoxifen may impact on the true changes in hormonal status under investigation, so therefore both were included in these criteria. As well, medications that promote weight gain or a co-morbid medical illness could have an impact on body weight by mechanisms other than those related to adjuvant chemotherapy. However, the use of dexamethadone was permitted, despite the impact of the drug on weight gain, since it is a powerful and effective common anti-nausea drug and a standard of care in this population.

\section{Measurements}

The measurements performed in this study were chosen after careful consideration of accuracy, reliability, validity and feasibility.

Body weight was measured using a standard scale to the nearest $0.5 \mathrm{lb}$ and converted to kilograms. Subjects were clothed, but without shoes. 
Body composition was measured using the current gold standard measure, Dual-energy x-ray absorptiometry (DXA) ${ }^{6}$. This instrument measures bone mineral mass, fat mass, and DXA lean body mass. All scans were acquired on a Hologic QDR 4500W densitometer (Hologic Inc., Waltham, MA) by a single trained and certified densitometrist. Mandatory daily Hologic quality control procedures and body composition calibration were completed using the anthropomorphic spine and step phantoms provided by the manufacturers. Analysis was completed according to Hologic guidelines, using software version V8.26a:5

Menstrual status was determined by a questionnaire, which asked about menstrual pattern in the previous year, and a menstrual calendar log sheet completed during treatment, on which participants noted the start and finish of their menstrual periods.

Resting metabolic rate was determined by indirect calorimetry using the $\mathrm{K} 4 \mathrm{~b}^{2}$ portable metabolic cart (Cosmed, Rome, Italy). Indirect calorimetry measures the oxygen $\left(\mathrm{VO}_{2}\right)$ consumed and carbon dioxide $\left(\mathrm{VCO}_{2}\right)$ produced by the lungs, which normally equals that used and released by the body tissues 7 , 8. Caloric expenditure can then be estimated by measuring respiratory gases, and for this study was converted into energy expenditure in kilocalories used per day (kcal/day). This measurement was completed by a trained operator at the participant's home in the morning, shortly after the participant awoke.

Participants were instructed to fast for 12 hours preceding measurement, and drink only water. These measures were not coordinated with menstrual cycle 
due to specific test timing in relation to chemotherapy delivery and the possible development of amenorrhea. In a quiet, comfortable place, participants were asked to lie quietly for 25 minutes. This allowed for a period of 5 minutes to return the participant to the resting levels, and 20 minutes of data to be used to ascertain resting metabolic rate. Resting metabolic rate was calculated by averaging the breath-by-breath $\mathrm{kcal} /$ day data provided by the $\mathrm{K} 4 \mathrm{~b}^{2}$ to determine energy expenditure $(\mathrm{EE})$, using the equation provided by the manufacturer:

$\mathrm{EE}(\mathrm{kcal} / \mathrm{min})=3.781 \times \mathrm{VO}_{2}+1.237 \times \mathrm{VO}_{2}, \quad$ if $\mathrm{UN}$ (ureic nitrogen) $=0$ From this kcal/day was calculated using:

$$
\mathrm{EE}(\mathrm{kcal} / \text { day })=\mathrm{EE}(\mathrm{kcal} / \mathrm{min}) * 1440
$$

These equations produced an estimate of energy expenditure which was similar to that calculated using a value $4.90 \mathrm{kcal} / \mathrm{L} \mathrm{O}_{2}$ of energy released from a mixed fuel source (carbohydrate and fat) multiplied by $\mathrm{VO}_{2} \cdot \mathrm{L} / \mathrm{min}^{9}$. To avoid computation errors, the values provided by the $\mathrm{K} 4 \mathrm{~b}^{2}$ were used.

\section{Logistics}

The project was approved by the Breast Tumour Group of BCCA, and received ethical approval from Research Services at the University of British Columbia. Oncologists and nursing clinicians at the VCC of BCCA performed the initial recruitment of subjects from the new patient clinical practice. A onepage overview of the study was provided to these clinicians, and recruitment flyers were provided for the new patient clinic. If interest was expressed on the part of a new patient, the investigators provided a description of the study purpose and design over the telephone. At this time, investigators assessed the 
potential participant for inclusion in the study based on the inclusion and exclusion criteria. If the participant was appropriate for the study and wished to proceed, an initial visit to the participant's home allowed for the measurement of weight and resting metabolic rate, and for the administration of the menstrual status questionnaire prior to commencement of chemotherapy. Body composition using the DXA scan was then performed prior to commencement of chemotherapy, at the University of British Columbia.

Once adjuvant chemotherapy commenced, body weight and resting metabolic rate were measured during chemotherapy treatment. Measurement occurred once per cycle of chemotherapy within 7 days of the last dose. Participants underwent two main types of chemotherapy, cyclophosphamide, epirubicin, and 5-fluorouracil (CEF) or doxorubicin (Adriamycin) and cyclophosphamide (AC). CEF involved six 4-week cycles, with intravenous chemotherapy on day 1 and 8 , and oral chemotherapy taken from days 2-7 and days $9-14$, followed by a 14 day "rest" period ${ }^{10}$. Measurements were completed between days $15-22$, and with the pre-assessment amounted to a total of seven measurements. With AC, chemotherapy was delivered intravenously on day 1 and repeated every 21 days for four cycles ${ }^{10}$. With this regime, measurements were done between days $2-8$, for a total of five measurements. The measurement of body composition was then repeated within one week of the completion of chemotherapy. 
Statistical Analysis

Descriptive statistics on each participant's age, height, weight, body mass index, and body fat percentage were calculated. A one-way repeated measures ANOVA, with five levels (for all participants) and seven levels (for those undergoing CEF) of the dependent measure, was used to analyze change in weight and resting metabolic rate. Directional change was analyzed by individual linear regression analysis, which identified slope and intercept points for all participants. A one-sample t-test was then conducted to ascertain if the combined slope of the individual lines was significantly different from zero. The accepted level of significance was set at $p<0.05$. Body composition was analyzed using a repeated measure t-test for each total and regional value of lean mass, fat mass, and percent body fat. While this type of analysis increased the chance of Type I error, the low number of participants in the study did not allow for other types of analysis, such as MANOVA. To combat Type I error, an alpha level of 0.01 was used due to the number of planned comparison. All analyses were conducted using Statistical Package for the Social Sciences (SPSS, 1995, version 6.1, SPSS Inc, Chicago, Illinois). 


\section{Chapter 3 - RESULTS}

Twelve women were identified as potential participants by the VCC staff. Of these, five agreed to participate, and provided written consent. The participants had a mean age of 49.2 years (SD 5.4, range $=40-54$ ), a mean height of $166.9 \mathrm{~cm}(\mathrm{SD} 7.3$, range $=157.5-176.0)$, a mean initial weight of 75.2 $\mathrm{kg}$ (SD 17.8, range $=57.7-101.2)$, a mean initial body mass index (BMI) of 27.5 $($ SD 8.8, range $=20.3-40.8)$, and mean initial body fat of $34.5 \%($ SD 8.9 , range $=$ 26.2-46.9) (see raw data in Appendix D). Four were pre-menopausal, and one was menopausal.

Following surgical excision of the breast tumour(s), the subsequent chemotherapy protocol was determined by patient-physician consultations. Individual pathology and treatment regimes are summarized in Table 1 and 2 , respectively.

Decisions of particular chemotherapy drug protocols were made in consideration of many factors, such as pathology results and potential side effects. Participant 3 had a 22 day (as opposed to 14 day) delay between the first and second CEF treatments due to low white blood cell counts, and was eventually put on a longer cycle with 14 days of treatment and 21 days of rest for the last four cycles. Otherwise all participants continued with the described protocols. 
Table 1. Surgical results with pathology and staging results of cancer

\begin{tabular}{c|ccccc}
\hline Participant & Surgery & $\begin{array}{l}\text { Nodes } \\
\text { removed } \\
\text { (positive } \\
\text { nodes) }\end{array}$ & Type & Stage & $\begin{array}{c}\text { ER } \\
\text { Status }\end{array}$ \\
\hline 1 & $\begin{array}{c}\text { L } \\
17(9)\end{array}$ & IFDC & T2N1aM0 & + \\
3 & $\begin{array}{c}\text { Iumpectomy } \\
\text { R partial } \\
\text { mastectomy } \\
\mathrm{R}\end{array}$ & $7(1)$ & IFDC & T3N1M0 & + \\
4 & $\begin{array}{c}\text { mastectomy } \\
\text { L partial } \\
\text { mastectomy } \\
\text { L }\end{array}$ & $10(0)$ & IFDC & T2NOM0 & - \\
\hline mastectomy & $4(0)$ & IFDC/DCIS & T2NOM0 & + \\
\hline
\end{tabular}

$\mathrm{R}=$ right; $\mathrm{L}=$ left; \# Nodes removed = axillary lymph nodes removed during surgery; \# Positive = number of axillary lymph nodes positive for carcinoma; DCIS = ductal carcinoma in situ; IFDC = infiltrating ductal carcinoma; Stage = see Appendix B for information about pathology and staging; ER Status = estrogen receptor status

Table 2. Chemotherapy regimes

\begin{tabular}{c|ccc}
\hline Participant & Treatment & Cycle Length (weeks) & \# of Treatments \\
\hline 1 & CEF & 4 & 6 \\
2 & CEF & 4 & 6 \\
3 & CEF & $4 / 5$ & 6 \\
4 & AC & 3 & 4 \\
5 & AC & 3 & 4 \\
\hline
\end{tabular}

CEF = cyclophosphamide, epirubicin, 5-fluorouracil (CEF); $A C=$ doxorubicin (Adriamycin) and cyclophosphamide

\section{Testing}

Resting metabolic rate and body weight were measured within one week of the end of each treatment cycle, with some exceptions due to timing conflicts for the portable metabolic equipment which was also used for another research study being conducted out of town. The fourth measurement for participant 2 was completed on the $26^{\text {th }}$ day of the cycle and on the $22^{\text {nd }}$ day for participant 3 . Participant 4 had the final measurement nine days after treatment 
administration, and participant 5 had the measurement at the first $A C$ treatment, nine days later. Calibration problems with the portable metabolic cart led to exclusion of the fifth measurement for participant 1 and the last measurement of participant 5 from the data analysis.

\section{Weight Change}

Weight was not significantly different across time for all participants $(p=$ $0.16)$ or when only those receiving CEF were considered $(p=0.29)$. A plot of means shows a non-significant increase in weight over time (see Fig 1a and 1b). However, further analysis of directional change showed that the combined slope of individual regression lines was not different from zero $(p=.132)$. Table 3 shows individual weight data and change scores. 
Fig. 1a - Weight Main Effect

$F(4,16)=1.88 ; p<.16$

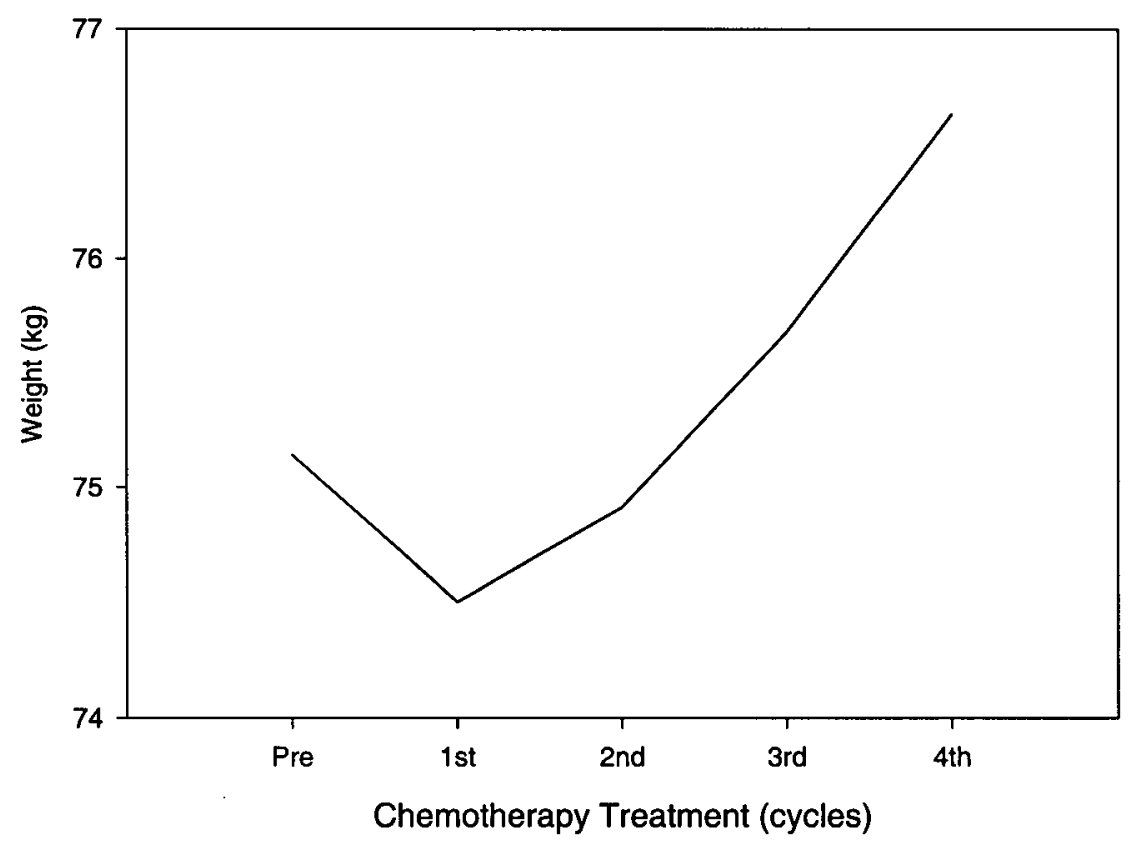

Fig. 1b - Weight Main Effect (CEF only) $F(6,12)=1.39 ; p<.29$

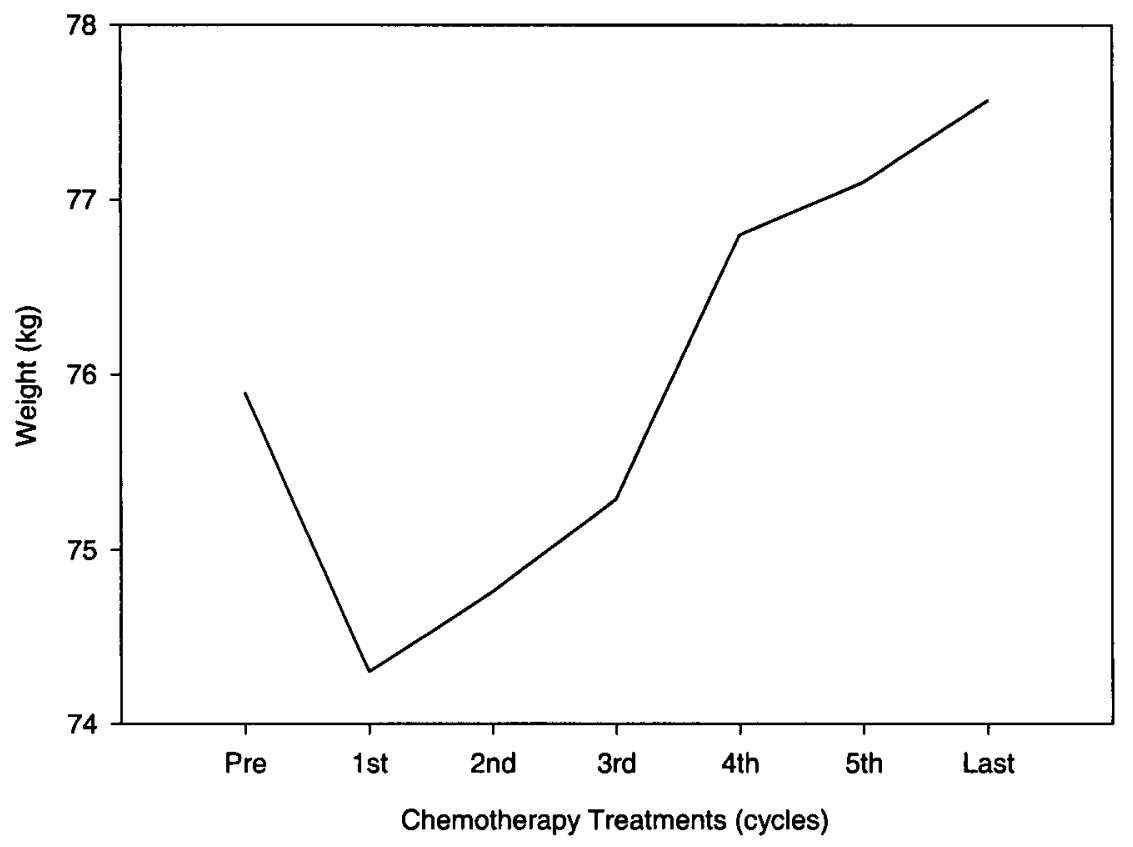


Table 3 - Weight before and at the end of treatment $(\mathrm{kg})$, plus change $(\mathrm{kg})$

\begin{tabular}{c|ccc}
\hline Participant & Starting weight & End weight & Weight change \\
\hline 1 & 101.0 & 98.29 & -2.71 \\
2 & 69.01 & 75.82 & 6.81 \\
3 & 57.66 & 58.6 & 0.94 \\
4 & 85.13 & 86.03 & 0.90 \\
5 & 62.88 & 66.73 & 3.85 \\
\hline
\end{tabular}

\section{Resting Metabolic Rate}

Resting metabolic rate did not change significantly over time for all participants $(p=0.323)$ or only for those undergoing CEF $(p=0.314)$. A plot of the mean effect (see Fig. $2 a$ and $2 b$ ) shows a decrease across time after the third and fourth treatments (all participants), and a consistent decrease after the first treatment, but an increase after the final treatment, in those who underwent six treatments of CEF. Table 4 contains mean resting metabolic rate data. Analysis of directional change found that the combined slope of individual regression lines was not different from zero $(p=0.594)$. However, plots of individual resting metabolic rate data show fluctuations over time but a return to near pre-chemotherapy values at the completion of treatment (see Figure 3). 
Fig 2a-Resting Metabolic Rate Main Effect

$F(4,12)=1.31 ; p<.32$

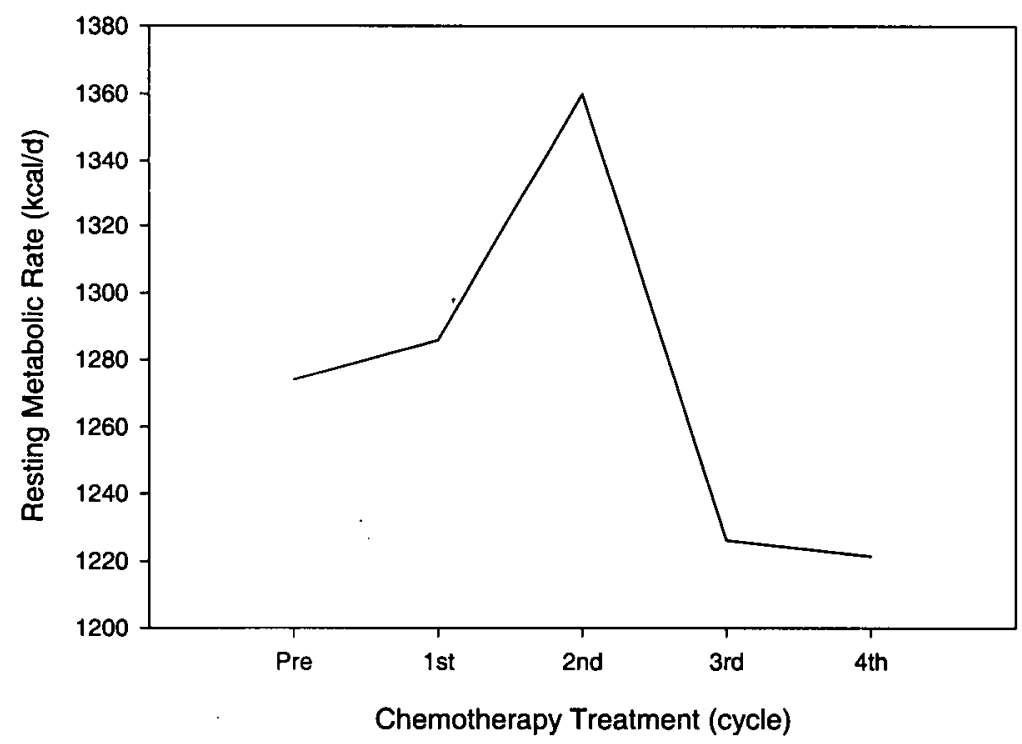

b. Resting Metabolic Rate Main Effect (CEF only) $F(6.6)=1.51 ; p<.31$

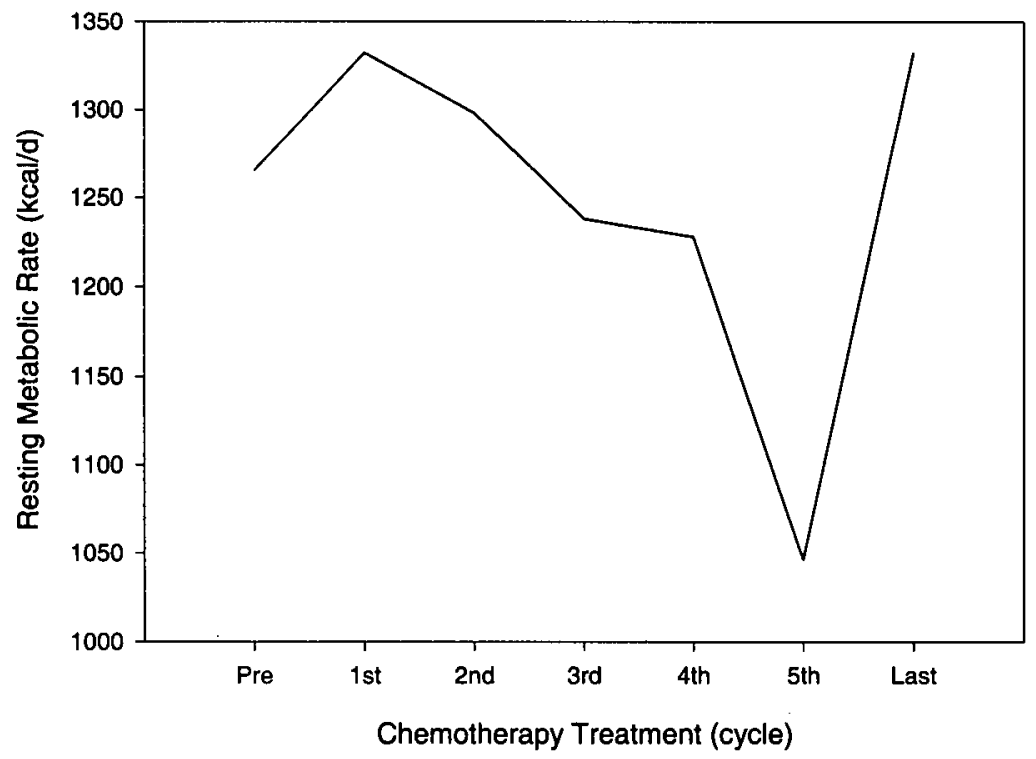


Table 4 - Mean resting metabolic rate measures across treatment time in $\mathrm{kcal} /$ day (standard deviation)

\begin{tabular}{c|ccccccc}
\hline Participant & Pre- & First & Second & Third & Fourth & Fifth & Sixth \\
\hline All & 1274 & 1286 & 1360 & 1226 & 1222 & & \\
& $(159)$ & $(259)$ & $(201)$ & $(66)$ & $(185)$ & & \\
& 1266 & 1332 & 1298 & 1238 & 1228 & 1046 & 1332 \\
CEF & $(204)$ & $(158)$ & $(190)$ & $(90)$ & $(225)$ & $(104)$ & $(83)$ \\
& & & & & & & \\
\hline
\end{tabular}

Figure 3 - Resting Metabolic Rate for all participants over treatment time

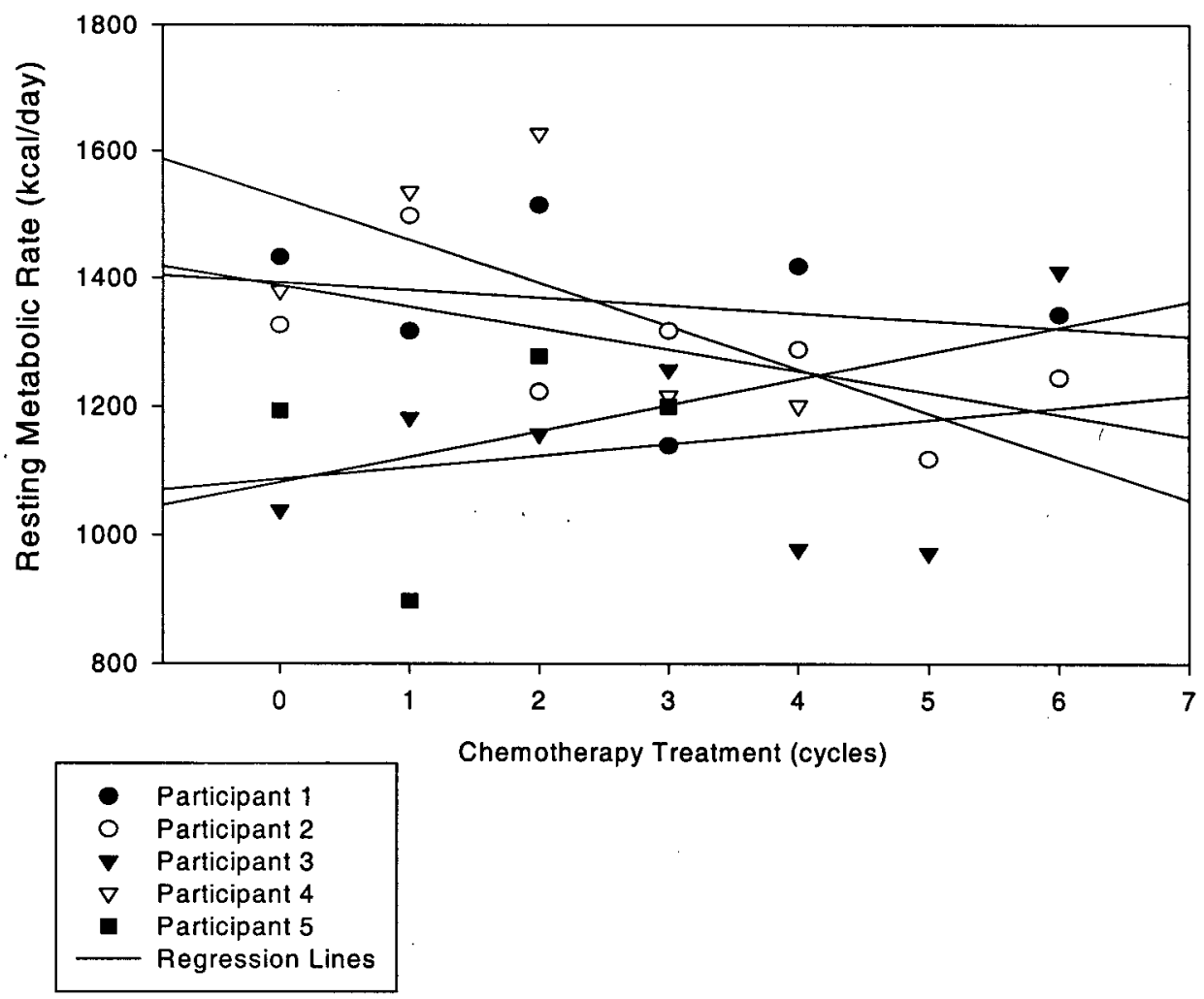




\section{Body Composition}

Total body composition and regional body composition changes were analyzed (see Table 5). Total fat mass $(p=0.185)$, lean mass $(p=0.807)$ and percent fat mass $(p=0.095)$ did not significantly change. Several regional measures, namely a decrease in left arm lean mass $(p=0.041)$, increase in trunk percent fat $(p=0.059)$, increase in left leg lean mass $(p=0.078)$ and an increase in left leg total mass $(p=0.061)$, approached significance.

Table 5 - Measures of total fat mass, lean mass, and percent body fat before and after treatment and for those areas approaching significance * (standard deviation), using DXA (g).

\begin{tabular}{c|ccccc}
\hline Tissue & Pre-treatment & Post-treatment & Change & p value & $\begin{array}{c}\% \\
\text { change }\end{array}$ \\
\hline Fat mass & $26879.4(13157.0)$ & $28965.8(11115.4)$ & +2086.4 & .185 & $+7.2 \%$ \\
Lean mass & $45256.3(4259.2)$ & $45165.5(4808.7)$ & -90.8 & .807 & $-0.2 \%$ \\
$\%$ Fat & $34.3(8.8)$ & $36.8(6.8)$ & $+2.5^{\star}$ & .095 & $+6.8 \%$ \\
$\begin{array}{c}\text { L arm lean } \\
\text { mass }\end{array}$ & $2008.8(345.0)$ & $1929.6(328.9)$ & $-79.2^{\star}$ & .041 & $-4.1 \%$ \\
$\begin{array}{c}\text { Trunk \% fat } \\
\text { mass } \\
\text { L leg lean } \\
\text { mass } \\
\text { L leg total } \\
\text { mass }\end{array}$ & $32.1(11.1)$ & $35.6(8.9)$ & $+3.5^{\star}$ & .059 & $+9.8 \%$ \\
\hline $\mathrm{L}=$ left & $12435.0(2572.4)$ & $12743.7(2436.8)$ & $+308.7^{\star}$ & .061 & $+2.4 \%$ \\
\hline
\end{tabular}




\section{Menstrual Status}

Menstrual status was reported by participants prior to the start of chemotherapy and the presence of menstrual activity was recorded on a calendar throughout treatment. The data are presented in Table 6 as either yes or no, to the question of a menstrual period after each cycle of chemotherapy for those on CEF (duration of cycle equals four weeks). The three week cycle of AC did not necessarily allow for one menstrual period per cycle (based on a 28 day menstrual cycle), however, of the two participants undergoing $A C$, one was postmenopausal prior to treatment and the other became amenorrheic after the fourth treatment.

Table 6 - Presence of a menstrual period throughout treatment.

\begin{tabular}{l|lcccccc}
\hline Participant & Prior & First & Second & Third & Fourth & Fifth & Sixth \\
\hline 1 & Perimenopausal & $\mathrm{N}$ & $\mathrm{N}$ & $\mathrm{N}$ & $\mathrm{N}$ & $\mathrm{N}$ & $\mathrm{N}$ \\
& (every 3 months) & & & & & & \\
2 & Regular & $\mathrm{Y}$ & $\mathrm{Y}$ & $\mathrm{N}$ & $\mathrm{N}$ & $\mathrm{N}$ & $\mathrm{N}$ \\
3 & Regular & $\mathrm{Y}$ & $\mathrm{Y}$ & $\mathrm{N}$ & $\mathrm{N}$ & $\mathrm{N}$ & $\mathrm{N}$ \\
4 & Menopausal & $\mathrm{N}$ & $\mathrm{N}$ & $\mathrm{N}$ & $\mathrm{N}$ & $/$ & $/$ \\
5 & Regular & $\mathrm{Y}$ & $\mathrm{Y}$ & $\mathrm{N}$ & $\mathrm{N}$ & $/$ & $/$ \\
\hline $\mathrm{N}=$ no menstrual period; $\mathrm{Y}=$ menstrual period; / = did not receive a fifth or sixth \\
cycle
\end{tabular}




\section{Chapter 4 - DISCUSSION}

This investigation examined weight gain in women who were undergoing adjuvant chemotherapy as part of breast cancer treatment, as well as three factors associated with weight gain, namely resting metabolic rate, body composition and menstrual status. While no significant changes were noted in weight or the measured associated factors, the data show interesting results.

Significant weight gain was not observed in this sample. This is in agreement with two recent studies 5,11 . One reason may be that participants in this study received chemotherapy protocols that were shorter and more intensive courses than those that have been linked to weight gain in the past ${ }^{11-}$ 13. The mixture of both CEF and AC chemotherapy protocols may have also impacted the results, as the $\mathrm{AC}$ protocol uses fewer chemotherapy agents, shorter treatment duration and intravenous administration, all factors that have been associated with less weight gain $5,14,15$.

However, individual analysis highlights the fact that all participants except one did gain weight over a four to six month time frame. In a study of longitudinal body weight measurements taken 10 years apart, an average weight gain of $2 \mathrm{~kg}$ was seen in the elapsed time, for women 30 to 55 year of age 16 . Two participants gained half this amount (approximately $1 \mathrm{~kg}$ ) in the course of treatment and two more participants gained far more $(6.81$ and $3.85 \mathrm{~kg}$, respectively). Clearly there is a factor that is influencing weight change in these women beyond what is normally seen in women of the same age. Also of note

is the observation of Rock et al. ${ }^{15}$, that weight loss rather than weight gain, was 
seen in women who had a higher body mass index (BMI) before breast cancer diagnosis. Our data supports this as the individual with an initial BMI of 40.8 lost $2.71 \mathrm{~kg}$, while the other participants who had lower BMI values ranging from 20.3 to 32.0 , all gained weight.

The duration of observation in this investigation may have contributed to our findings, as there is evidence to suggest that the observed weight gain is not limited to the treatment time. Despite seeing no weight gain during chemotherapy treatment, both Demark-Wahnefried et al. 11 and Kutynec et al. 5 noted weight gain at one year follow up. Levine et al. 17 noted a gain of $1.8 \mathrm{~kg}$ in a sample of 32 women during treatment and a mean weight gain of $6.03 \mathrm{~kg}$ at two years post treatment. It is plausible that if weight gain is the result of a change in body composition, namely a decrease in lean body mass and, in turn, resting metabolic rate, that these changes take time to result in noticeable change in weight. Perhaps the duration of measurement was not long enough to capture potential weight change in our sample.

This investigation measured resting metabolic rate at more time points than any previous study and improved on previous research methods by measuring resting metabolic rate at the participant's home to avoid the inevitable increase in energy expenditure that occurs with traveling to test sites. The portable metabolic cart used, the COSMED K4b ${ }^{2}$, has been validated in our lab and by other investigators 18,19 . The data from this investigation did not show a significant change in resting metabolic rate across time, consistent with the findings of two recent studies, Kutynec et al. and Demark-Wahnefried et al. 3, 
5. However, plots of the means appear to show a decreasing trend past the mid-point of treatment for all participants, that returns close to pre-treatment values after the last treatment in those who received CEF (see Figure $2 \mathrm{a}$ and 2b). These findings correspond with those of Demark-Wahnefried et al. ${ }^{11}$, who noted a significant decrease of resting metabolic rate at midpoint of treatment $(1277 \pm 213 \mathrm{kcal})$ that then returned to levels similar to baseline by the end of treatment (baseline $1354 \pm 213 \mathrm{kcal}$ to $1325 \pm 232 \mathrm{kcal}$ ). These investigators also found a decrease in lean body mass, which was used to explain the midpoint decrease, but noted that this did not explain the metabolic rate rebound at the end of treatment. The resting metabolic rate values recorded in this study were comparable to those noted in previous studies 5,11 .

If change in resting metabolic rate in women undergoing adjuvant chemotherapy for breast cancer does indeed occur, it seems to be a plausible explanation for weight gain. A decrease in resting metabolic rate has been associated with cell death from chemotherapy, body composition changes and with a decrease in energy expenditure in the form of physical activity ${ }^{14}$. On closer examination of these factors, the extent of change in resting metabolic rate as a result of cell death due to chemotherapy treatment has not been established, and the extent of lean body mass loss needed to impact on resting metabolic rate is another important consideration. Changes in lean and body fat mass, related to shifts in hormonal milieu and menopausal status, has been well documented 20, 21. In the general population, a curvilinear decline in RMR with age was noted in women age 51-81 years, which was primarily associated with 
a similar curvilinear decrease in lean body mass 20 . A longitudinal comparison of premenopausal women found that women who experience menopause had "an accelerated loss of fat-free mass, and increased central adiposity", along with an increase in total fat mass, and a greater decrease in resting metabolic rate, than those who remained premenopausal ${ }^{21}$. A decrease in physical activity during treatment has also been implicated 3,11 . A shift towards greater energy intake than expenditure is one explanation for the weight gain, while a loss of lean body mass due to reduced activity and muscle use, which in turn reduces resting metabolic rate, is another possible explanation. Rock et al. 15 found that women undergoing chemotherapy treatment who engaged in walking or strenuous exercise had less weight gain than those who did not engage in these types of activities. No measure of body composition was used in this study. However, the loss of lean body mass appears to be an important factor. A study comparing postmenopausal women, without cancer, age 49 to 70 years who were "long-term exercisers" to "long-term non-exercisers", found that absolute and per kilogram of body weight resting metabolic rate was significantly higher in the more active group, which investigators felt was due to the greater percent of lean body mass noted in the active group 22 .

Our sample did not have any significant body composition changes, however, a gain in trunk percent fat and total body percent fat approached significance, along with a gain in left leg lean mass and a loss of left arm lean mass. Regional analysis for lean and fat tissues, using DXA, is less precise than that for total measures and requires a larger sample size to evaluate 
changes 23 . Therefore, the changes noted in the trunk and extremities must be viewed with caution due to the sample size used in this study. Body hydration also has an impact of DXA lean body mass results 24 . If there is a potential increase in body water with treatment, in the form of edema or water retention, this may result in an increase in the amount of lean body mass recorded, but mask a possible loss of muscle tissue.

The development of "sarcopenic obesity", defined as weight gain without gains in lean body mass, has been recently proposed by Demark-Wahnefried et al. 3. These investigators found a mean weight gain of $2.1 \mathrm{~kg}$ in this population, with an increase in percent body fat, and total fat mass, and a decrease in total and leg lean body mass using DXA. The concurrent decrease in recorded physical activity was the main mechanism proposed for both weight gain and body composition changes. Kutynec et al. 5 , using DXA, noted a similar pattern of change in total and leg lean body mass, and an increase in percent body fat, but no change in physical activity level and only a small increase in resting energy expenditure was noted. The authors suggested that the increase in resting metabolic rate at the end of treatment was an attempt by the body to return metabolic rate to pre-treatment levels.

Reliability of the measures of lean mass, fat mass and \% fat by the DXA scan used in this investigation is presented in Appendix $\mathrm{C}$. The precision of total body measures for women as a coefficient of variation (lean mass $=0.36 \%$, fat mass $=1.33 \%$, and percent body fat $=1.42 \%$ ) are higher than that for regional measures (lean mass $=0.91-2.58 \%$, fat mass $=2.55-5.87 \%$, and percent body 
fat $=2.48-5.82 \%)$. However, these values are consistent with published reliability studies 23,25 . Values calculated from the data presented in the recent study by Demark-Wahnefried ${ }^{3}$ with 36 participants, show a $1.4 \%$ decrease in leg lean mass and a $1.8 \%$ decrease in trunk lean mass. Using the sample sizes provided by Chilibeck et al. 23,21 participants are required to show a $2 \%$ change in leg lean mass and trunk lean mass. To show a $1 \%$ change left leg lean mass, 91 participants are needed, and 83 participants are needed to show the same change in trunk lean mass. For a $5 \%$ change in total fat mass to be demonstrated, a sample size of 5 is required, while 17 participants are need to demonstrate a $5 \%$ change in trunk fat mass 23 . A $7.2 \%$ increase in total fat mass and $6.8 \%$ increase in total body fat percentage was reported in this investigation, using a sample size of 5 . A $9.8 \%$ increase in trunk $\%$ fat mass was also noted.

Menstrual status appears to be a significant factor in determining weight gain in this population. Natural menopause is thought to lead to a loss in lean body mass, increase in total body fat and altered fat distribution, and it has been proposed that these changes are accentuated and more rapid in women undergoing premature menopause, such as that which is seen with adjuvant chemotherapy treatment $1,3,12,14,21$. In this investigation those undergoing CEF all developed chemotherapy induced amenorrhea. One participant who was perimenopausal prior to start of CEF chemotherapy, having menstrual periods approximately every three months for the two years prior, became amenorrheic with the first dose of CEF chemotherapy and actually lost weight (- 
$2.71 \mathrm{~kg}$ ) during treatment. However, of those with previously regular menstrual cycles, the two participants who received CEF became amenorrheic after two cycles of chemotherapy and gained $6.81 \mathrm{~kg}$ and $0.94 \mathrm{~kg}$ respectively. Of those who underwent $A C$, the individual that was postmenopausal prior to diagnosis and treatment, gained only $0.9 \mathrm{~kg}$, and the other, who was the youngest participant in the study, became amenorrheic after the fourth treatment and gained $3.85 \mathrm{~kg}$. These observations lend credence to the hypothesis that weight gain is the result of accelerated weight changes normally associated with menopause. Goodwin et al. 26 found that adjuvant treatment and the onset of menopause were the strongest clinical predictors of weight gain. The same investigators found that age and use of systemic chemotherapy were the most important predictors of menopausal onset in premenopausal women being treated for breast cancer, with a $>40 \%$ risk in 40 year old women and close to $100 \%$ in 50 year old women 27.

\section{Summary of individualized results:}

Overall the mean weight gain in our study was $1.9 \mathrm{~kg}$ (range $=-2.71$ to $6.81 \mathrm{~kg}$ ), with all but one participant gaining weight. Individual data shows important specific aspects of this question. Participant 1 actually lost weight, but this was consistent with previous findings that those who started out at a heavier weight, did indeed tend to lose weight 15 . Participant 2 and 3 both become menopausal and showed a trend towards a lower resting metabolic ráte until a rebound at the end of treatment. Together they serve as an example of the range of weight gain seen in this population, with participant 2 gaining a 
significant amount of weight $(6.81 \mathrm{~kg})$ and participant 3 gaining much less $(0.94$ $\mathrm{kg}$ ). Participant 4 serves as an example of those women who are postmenopausal prior to treatment, and experience little to no change in weight or resting metabolic rate. Participant 5 received a shorter and less intense chemotherapy regime (AC) and became menopausal, but gained less weight than another study participant who underwent the longer CEF treatment.

\section{Summary:}

While statistical analysis did not result in significant findings, a trend toward weight gain was observed. The most striking associated finding was the development of chemotherapy-induced amenorrhea in all subjects who were premenopausal prior to treatment. There was a trend toward a decrease in resting metabolic rate during treatment that may return to pre-treatment levels after six cycles of chemotherapy. An increase in total fat mass, total percent body fat, and trunk percent body mass, was also noted. These changes in weight, resting metabolic rate, and body composition, which are associated with natural menopause, may be accelerated in women who develop chemotherapyinduced amenorrhea.

\section{Limitations:}

By far the greatest limitation to this investigation is the low number of participants $(n=5)$. Conclusions are also hampered by the heterogeneity of the participants' initial characteristics (weight, BMI, height, age, body composition, resting metabolic rate, and treatment type). The small number of subjects and between-subject variance makes the identification of real change difficult. While 
there were trends towards weight gain, a decrease in resting metabolic rate, and increase in trunk fat mass and total percent fat, these cannot be proven by statistical methods due to the above considerations. If more participants could be included in the analysis, the change in the outcome of the measured variables remains to be seen. This investigation only focused on one potential mechanism of weight gain, namely changes in body composition and resting metabolic rate, which may be associated with chemotherapy-induced amenorrhea. The impact of physical activity and dietary intake on energy balance was not assessed. These factors have been shown to contribute to the weight gain in this population. However, the inherent problem of the self-report data gathered with the feasible measurement tools for both physical activity and dietary intake, makes establishing validity difficult. This investigation concentrated on using accurate measurement tools and improved on their previous use, while focusing on only one proposed cause of weight gain in this population.

\section{Future Recommendations:}

Follow up measures of weight, resting metabolic rate and body composition at one year post treatment would allow for the capture of potential post treatment weight gain that has been observed by others. The addition of reliable measures of dietary intake and physical activity would allow for the impact of overall energy balance to be better elucidated. The timing of such measures could also be important, if the reported side effects of chemotherapy treatment, such as nausea and fatigue that greatly affect these measures, 
change over the treatment cycle. The tie between resting metabolic rate and body composition should also be further investigated, for if a loss in lean body mass is contributing to a significant decrease in resting metabolic rate, an intervention that attempts to maintain or increase lean body mass, may be able to prevent significant weight gain. 


\section{REFERENCES}

1. Demark-Wahnefried W, Rimer B, Winer E. Weight gain in women diagnosed with breast cancer. [Review]. Journal of the American Dietetic Association. 1997;97:519-26,529.

2. Knobf M, Mullen J, Xistris D, Moritz D. Weight gain in women with breast cancer receiving adjuvant chemotherapy. Oncology Nursing Forum. 1983;10:2833.

3. Demark-Wahnefried W, Peterson BL, Winer EP, Marks L, Aziz N, Marcom PK, et al. Changes in weight, body composition, and factors influencing energy balance amoung premenopausal breast cancer patients receiving adjuvant chemotherapy. Journal of Clinical Oncology. 2001;19:2381-2389.

4. Cheney CL, Mahloch J, Freeny P. Computerized tomography assessment of women with weight changes associated with adjuvant treatment for breast cancer. Am J Clin Nutr. 1997;66:141-6.

5. Kutynec CL, McCargar L, Barr SI, Hislop G. Energy balance in women with breast cancer during adjuvant treatment. Journal of the American Dietetic Assocation. 1999;99:1222-1227.

6. Kohrt W. Body Composition by DXA: tried and true? Med Sci Sport Exercise. 1995;27:1349-1353.

7. Murgatroyd PR, Shetty PS, Prentice AM. Techniques for the measurement of human energy expenditure: a practical guide. International Journal of Obesity \& Related Metabolic Disorders. 1993;17:549-568. 
8. Wilmore J, Costill D. Physiology of Sport and Exercise. Champaign Illinois: Human Kinetics; 1994.

9. Fox EL, Bowers RW, Foss ML. The Physiological Basis of Physical Education and Athletics. 4th ed. New York: Saunders College Publishing; 1988. 10. Perry M, editor. The Chemotherapy Source Book. 2 nd. ed. Baltimore: Williams \& Wilkins; 1996.

11. Demark-Wahnefried W, Hars V, Conaway M, Havlin K, Rimer B, McElveen G, et al. Reduced rates of metabolism and decreased physical activity in breast cancer patients receiving adjuvant chemotherapy. Am J Clin Nutr. 1997;65:1495-501.

12. Camoriano J, Loprinzi C, Ingle J, Therneau T, Krook J, Veeder M. Weight change in women treated with adjuvant therapy or observed following mastectomy for node-positive breast cancer. J Clin Oncol. 1990;8:1327-34.

13. Bonadonna G, Valagussa P, Rossi A. Ten-year experience with CMFbased adjuvant chemotherapy in resectable breast cancer. Breast Cancer Res Treat. 1985;5:95-115.

14. Demark-Wahnefried W, Winer E, Rimer B. Why women gain weight with adjuvant chemotherapy for breast cancer. [Review]. J Clin Oncol. 1993; 11:1418-29.

15. Rock C, Flatt S, Newman V, Caan B, Haan M, Stefanick M, et al. Factors associated with weight gain in women after diagnosis of breast cancer. Women's Healthy Eating and Living Study Group. Journal of the American Dietetic Association. 1999;99:1212-21. 
16. Williamson D. Descriptive epidemiology of body weight and weight change in U.S. adults. Annals of Internal Medicine. 1993;119:646-649.

17. Levine E, Raczynski J, Carpenter J. Weight gain with breast cancer adjuvant treatment. Cancer. 1991;67:1954-1959.

18. LaBreche J, McKenzie D. Evaluation of the COSMED K4b2 Portable Metabolic System during maximal exercise. Canadian Journal of Applied Physiololgy. 2001;26:492.

19. McLaughlin J, King GA, Howley ET, D.R. B, Ainsworth BE. Assessment of the Cosmed K4b2 portable metabolic system (Abstract). Med Sci Sport Exercise. 1999;31:S286.

20. Poehlman E, Goran M, Gardner A, Ades P, Arciero P, Katzman-Rooks S, et al. Determinants of decline in resting metabolic rate in aging females. $A m \mathrm{~J}$ Physiol. 1993;264:E450-E455.

21. Poehlman E, Toth M, Gardner A. Changes in energy balance and body composition at menopause: a controlled longitudinal study. Annals of Internal Medicine. 1995;123:673-675.

22. Withers R, Smith D, Tucker R, Brinkman M, Clark D. Energy metabolism in sedentary and active 49- to 70-yr old women. J Appl Physiol. 1998;84:13331340.

23. Chilibeck P, Calder A, Sale D, Webber C. Reproducibility of dual-energy x-ray absorptiometry. Canadian Association of Radiologists Journal. 1994;45:297-302. 
24. Roubenoff R, Kehayias JJ, Dawson-Hughes B, Heymsfield SB. Use of dual-energy $\mathrm{x}$-ray absortiometry in body-composition studies: not yet a "gold standard". Am J Clin Nutr. 1993;58:589-591.

25. Lohman $T$, Harris $M$, Teixeira $P$, Weiss $L$. Assessing body composition and changes in body composition: another look at duel-energy x-ray absoptiometry. Annals of the New York Academy of Sciences. 2000;904:45-54. 26. Goodwin P, Ennis M, Pritchard K, McCready D, Koo J, Sidlofsky S, et al. Adjuvant treatment and onset of menopause predict weight gain after breast cancer diagnosis. J Clin Oncol. 1999;17:120-9.

27. Goodwin P, Ennis M, Pritchard K, Trudeau M, Hood N. Risk of menopause during the first year after breast cancer diagnosis. Journal of Clinical Oncology. 1999;17:2365+.

28. Society CC. 2001 Statistics. In:: Canadian Cancer Society; 2001.

29. Murphy G, Jr. WL, Jr. RL, editors. Clinical Oncology. 2 nd ed. Atlanta: American Cancer Society Inc.; 1995.

30. Olivotto I, Gelmon K, Kuusk U. Intelligent Patient Guide to Breast Cancer. 1 ed. Vancouver: Intelligent Patient Guide Ltd.; 1995.

31. Knobf MT. Symptoms and rehabilitation needs of patients with early stage breast cancer during primary therapy. Cancer. 1990;66:1392-401.

32. Goodwin P, Panzarella T, Boyd N. Weight gain in women with localized breast cancer--a descriptive study. Breast Cancer Research \& Treatment. 1988;11:59-66. 
33. Heasman K, Sutherland H, Campbell J, Elhakim T, Boyd N. Weight gain during adjuvant chemotherapy for breast cancer. Breast Cancer Res Treat. 1985;5:195-200.

34. Campbell K, Harris S. Physical activity in the primary prevention of estrogen-related cancers: Is it effective? Physiotherapy Canada. 2000.

35. McTiernan A, Ulrich C, Slate S, Potter J. Physical activity and cancer etiology: associations and mechanisms. Cancer Causes \& Control. 1998;9:487509.

36. Foltz A. Weight gain in stage II breast cancer patients: a study of five factors. Oncol Nurs Forum. 1985;3:21-26.

37. Hardman J, Limbird L, Gilman A, editors. Goodman and Gilman's The Pharmacological basis of therapeutics. 10th ed. New York: McGraw-Hill; 2001. 38. Ganz PA, Schag CC, Polinsky ML, Heinrich RL, Flack VF. Rehabilitation needs and breast cancer: the first month after primary therapy. Breast Cancer Research \& Treatment. 1987;10:243-53.

39. Shapiro C, Recht A. Side effects of adjuvant treatment of breast cancer. New Eng J Med. 2001;344:1997-2008.

40. Grindel C, Cahill C, Walker M. Food intake of women with breast cancer during thier first six months of chemotherapy. Oncol Nurs Forum. 1989;15:447450.

41. Winningham ML, MacVicar MG. The effect of aerobic exercise on patient reports of nausea. Oncol Nurs Forum. 1988;15:447-50. 
42. Loprinzi C, Athmann L, Kardinal C. Randomized trial of dietician counseling to try to prevent weight gain associated with breast cancer adjuvant chemotherapy. Oncology. 1996;53:228-232.

43. Bines J, Oleske D, Cobleigh M. Ovarian function in premenopausal women treated with adjuvant chemotherapy for breast cancer. Journal of Clinical Oncology. 1996;14:1718-1729.

44. Mastro LD, Venturini M, Setoli M. Amenorrhea induced by adjuvant chemotherapy in early breast cancer patients: Prognostic role and clinical implications. Breast Cancer Res Treat. 1997;43:183-190.

45. Aslani A, Smith R, Allen B, Pavlakis N, Levi J. Changes in body composition during breast cancer chemotherapy with the CMF-regime. Breast Cancer Res Treat. 1999;57:285-290.

46. Guo S, Zeller C, Chumlea W. Aging, body composition and lifestyle: The Fels Longitudinal Study. Am J Clin Nutr. 1991;70:405-411.

47. Hagan RD. Benefits of aerobic conditioning and diet for overweight adults. Sports Med. 1988;5:144-55.

48. Leenders NYJM, Sherman WM, Nagaraja HN. Comparisons of four methods of estimating physical activity in adult women. Med Sci Sport Exercise. 2000;32:1320-1326. 


\section{APPENDIX A - Literature Review}

\section{Breast cancer demographics and current treatment}

To understand the importance of these observations it is necessary to look at the scope of breast cancer incidence and treatment. In 2001 it is estimated that 19,500 Canadian women will be diagnosed with breast cancer, and 5,500 will die from it ${ }^{28}$. Breast cancer is divided into stages I-IV, related to size of the tumor, number of involved axillary lymph nodes, and extent of metastases. The current method of treatment for stage I-III breast cancer most commonly involves surgical removal of the tumor, followed by adjuvant, meaning assisting or aiding, therapy used to reduce or eradicate any possible metastatic cancer cells. It is thought that adjuvant chemotherapy or ovarian ablation given to premenopausal, or adjuvant tamoxifen given to postmenopausal women, will reduce the odds of death by approximately $25 \%$ annually 29 . This allows for prolonged survival even if the patient is not cured of the disease.

The type of adjuvant therapy used varies related to several factors, such as size and node status of the cancer, and menopausal status of the woman 30 . A predisposition toward greater weight gain has been seen with an increased duration of treatment, with more weight gain observed with a year of treatment versus 6 months of treatment $13,14,26$. As well, undergoing any type of adjuvant therapy causes greater weight gain than none, but less weight gain is associated with the use of tamoxifen versus other chemotherapy 14,26 . The addition of prednisone is linked to even greater weight gain, and the route of 
chemotherapy delivery has also found to be contributory, with an oral regime resulting in greater weight gain than intravenous administration $14,31$.

\section{Sequelae of weight gain}

Weight gain during adjuvant therapy for breast cancer represents a health risk for the women involved. An increase in fat mass may predispose these women to other health problems, such as diabetes, heart disease, and orthopedic problems, such as osteoarthritis. Disease-free survival may also be affected. Camoriano et al. 12 found that premenopausal women who gained more weight than the median weight gain in the study $(5.9 \mathrm{~kg})$, had a risk of relapse 1.5 times greater, and a risk of death 1.6 times greater, than premenopausal women who gained less than the median weight. However, an association between weight gain and an increased risk of relapse has not been supported in other studies 32,33 . One aspect of primary breast cancer risk or risk of recurrence, is thought to be related to an interplay observed between an increased fat mass and the subsequent increased synthesis of estrogens and decreased amount of binding of estrogen to sex hormone binding globulins (SHBG). This results in overall higher amounts of circulating free estrogens 14 . This increased bioavailability and activity of estrogen is thought to be a prime factor in breast cancer development, and possible recurrence 1, 34, 35. The weight gain associated with breast cancer treatments may not necessarily result in clinically defined obesity, but rapid weight gain may induce a state of metabolic obesity, and potentiate the same hormonal effects 1 . 
Weight gain has also been found to be particularly distressing for women because it occurs at a time at which they may already be struggling with feelings of altered self image 1,26 . It is of note that, in one study, the majority of women who gained more than $4.5 \mathrm{~kg}$ reported this weight gain as distressing, and weight gain correlated to feelings of unhappiness, and anxiety over appearance 31. In another study, how to lose weight was ranked among the highest nutritional concerns of patients who had surgery for breast cancer, and only $27 \%$ of the group reported that they were satisfied with their current weight 15 .

\section{Postulated mechanism of weight gain}

Several factors have been identified as possible mechanisms to account for the weight gain seen in conjunction with adjuvant breast cancer therapy. Fundamentally, weight gain occurs in the presence of either increased energy intake, or decreased energy expenditure, or a combination of the two factors 36 . The main components of energy expenditure are resting metabolic rate, thermogenesis, and physical activity. Energy intake is measured from dietary intake. Changes in any one of these components of energy balance could shift the balance toward weight gain or loss. How the hormonal profile of women, which can be affected by adjuvant treatment, is thought to impact on energy balance is another important consideration, namely changes in menopausal status and other hormonal shifts. 


\section{A. Energy Balance}

\section{i) Resting Metabolic Rate}

By definition resting metabolic rate (RMR) is the energy expended by the body to maintain the essential physiological functions. RMR accounts for $60-$ $75 \%$ of the total energy we expend each day, and is directly related to fat-free mass ${ }^{8}$. Adjuvant chemotherapy has some potential avenues of impact on resting metabolic needs. Firstly, base energy requirements may be reduced due to the impact of chemotherapy on overall cell death 14 . Chemotherapy or antineoplastic agents work mainly by damaging the DNA of dividing cells, and targets those cells that are undergoing the most division, namely tumor cells, and normal tissues that proliferate rapidly (bone marrow, hair follicles, and intestinal epithelium). Exactly how this DNA damage causes cell death is not known. However, cellular responses of "cell-cycle arrest, DNA repair, and apoptosis, a specific form of nuclear fragmentation termed programmed cell death", have been noted 37 . There are several main categories of chemotherapy drugs, and combinations of drugs are commonly used to take advantage of synergistic biochemical interactions, overcome tumor resistance, and decrease overall toxicity 37 . Two common chemotherapy protocols used for breast cancer treatment are CEF and AC. The CEF protocol uses cyclophosphamide (alkylating agent), epirubicin (antibiotic), and 5-fluorouracil (antimetabolite). The AC protocol uses doxorubicin (Adriamycin) (antibiotic) and cyclophosphamide (alkylating agent). The alkylating agents, in this case cyclophosphamide, can act on cells at any stage in the division cycle by 
alkylation of the DNA. The actual mechanism of cell death that results is not well understood 37 . The antimetabolites used, such as 5-flurourcil, inhibit the synthesis of specific nucleotides or mimic other normal metabolites, which, in turn, interfere with synthesis or function of nucleic acids, specifically in RNA. Both doxorubicin and epirubicin are anthracycling antibiotics, and have several mechanisms of action. They are able to insert themselves into DNA, disrupting DNA and RNA synthesis, generate free radicals, which can attack DNA, and can interact with cell membranes and alter their function 37 . The desired result is tumor cell death, which may result in a decrease in overall resting energy requirements, as there are fewer cells requiring a supply of energy.

Secondly, chemotherapy can result in amenorrhea or induced menopause, thereby causing a change in hormonal milieu, and this in turn affects overall resting energy needs, either directly or indirectly by manifesting in changes in fat-free mass, and fat deposition and distribution 14 .

\section{ii) Thermogenesis}

Thermogenesis is defined as the energy expenditure above RMR that is not attributable to physical activity. It accounts for about $10 \%$ of total daily energy expenditure, and is induced by the likes of food intake and processing, exposure to cold, and emotional arousal 8,14 . The importance of thermogenesis on weight gain is controversial, since it only makes a small contribution to total daily energy expenditure. 


\section{iii) Physical Activity}

A study by Demark-Wahnefried et al. 11 found a $10 \%$ decrease in physical activity level from baseline to completion of treatment in women undergoing adjuvant chemotherapy. More recently, Demark-Wahnefried et al. 3 found a significantly greater mean energy output for physical activity in premenopausal women having only localized treatment (surgery and/or radiation) for breast cancer, compared to those receiving chemotherapy. In the absence of any other changes, a decrease in physical activity would result in a decrease in overall energy expenditure, and unbalance the energy intake and expenditure equation, thus resulting in possible weight gain. A decrease in physical activity also tends to lead to a decrease in lean body mass, thereby reducing RMR. The observed change in physical activity level is often attributed to two common side effects of adjuvant therapy, nausea and fatigue. The majority of women receiving adjuvant chemotherapy for breast cancer have mild to moderate nausea and vomiting, and fatigue has been reported in up to $96 \%$ of all patients who undergo such therapy 38,39 .

\section{iv) Dietary Intake}

Changes in dietary intake also impact energy balance. The results of research into changes in dietary intake with adjuvant breast cancer treatment range from no change, to increases or decreases in dietary intake 40,Foltz, 1985 \#149,Demark-Wahnefried, 1997 \#4. Research has alluded to increased cravings for sweet food and constant feelings of hunger in this population $1,14,36$. It has also been suggested that eating helps to relieve feelings of nausea $1,14,41$. 
While overeating has been proposed as a mechanism for weight gain in this population, intensive diet counseling on energy-restricted diets did not lead to significantly less weight gain when compared with those in the control arm of the study 42 . The interplay between dietary intake and physical activity is also key. If the level of energy expenditure decreases to the extent noted in the research, with respect to physical activity or RMR, and dietary intake is not adjusted for this decrease, overall weight gain would appear inevitable 21 .

\section{B. Hormonal factors}

\section{i) Menopausal Status}

Menopausal status of the women undergoing adjuvant chemotherapy appears to be a significant factor in weight gain. In a study by Camoriano et al. ${ }^{12}$, premenopausal women gained significantly more weight than postmenopausal women, with mean weight gains of $5.9 \mathrm{~kg}$ and $3.6 \mathrm{~kg}$, respectively. Several other studies echo these findings $2,26,33,36$. Adjuvant chemotherapy is suspected to decrease ovarian function, thereby inducing amenorrhea, which has been called chemotherapy-related amenorrhea 26,36 , 43. Decreased rate of metabolism, loss of lean body mass, propensity for increased fat accumulation, and altered fat distribution, are all changes associated with natural menopause 1,14, 21. Although unproven, it has been postulated that these normal changes are accentuated in women undergoing premature menopause, in this case from adjuvant chemotherapy 1,3 . The percentage of women undergoing menopause with polyagent chemotherapy was found to be from $53-89 \%$, with age and type of treatment being the 
strongest predictors 27,44 . Goodwin et al. 27 found the risk of undergoing menopause during chemotherapy began to increase at age 35 , and increased from there, with the majority of women age 45 and older becoming menopausal. Body size at baseline did not predict menopause in this study, but those women who gained larger amounts of weight during the first year after diagnosis, were the most likely to undergo menopause 27 . In a review by Bines et al., the median age that women became amenorrheic following adjuvant chemotherapy, varied from 38 to 46 years, compared to the average age of menopause in North American women of 50-52 years 43 . The median age for those who continued to menstruate regularly varied from 33 to 35 years 43 . In women less than 40 years, the median time to development of chemotherapy-related amenorrhea was from 6 to 16 months, and 2 to 4 months in women older than 40 years 43 . While some women may experience a return of menses following treatment, this was reported to occur in only $12-15 \%$ of women less 40 years old, and in less than $11 \%$ of women over 40 years of age 27,43 .

\section{ii) Hormonal Milieu}

Beyond changes in menstrual status, there are several ways in which adjuvant therapy is thought to influence changes in hormonal milieu in the body, and how these hormones, in turn, affect the body. Chemotherapy has been shown to cause fibrotic changes and follicular destruction in the ovaries, and cause reduced estradiol levels in premenopausal women. It has been suggested that estradiol is the principal ovarian hormone steroid affecting control 
of body weight, and that appetite changes and increased intake are associated with reduced serum estradiol levels 36 .

\section{Previous Investigations}

The basis of the investigation into the effects of adjuvant chemotherapy on body weight has been descriptive studies on weight changes and the type of treatment used. Measures of weight pre- and post-treatment, and at varied follow up periods, have been taken and compared with the type of treatment the participants have undergone.

Using this methodology, Knobf et al. ${ }^{2}$ found that $43.6 \%$ of women gained more than $4.5 \mathrm{~kg}$ and $28.7 \%$ gained in excess of $10 \%$ of their body weight over an average of 15 months. Hearman et al. 33 found that $96 \%$ of her group of 237 subjects gained weight during adjuvant therapy, with a mean increase of $4.3 \mathrm{~kg}$, and none lost weight in the 6 years of follow up. It was found that there was a striking association between the type of treatment received and the amount of weight gained. The group that received a combination of chemotherapy agents, with prednisone and ovarian ablation, gained the most weight (mean $=6.20 \mathrm{~kg}$, SD 3.56). The group who underwent a combination of chemotherapy agents, without prednisone, gained less (mean $=3.65 \mathrm{~kg}$, SD 3.39), with the group who received single agent chemotherapy gaining the least (mean $=2.72 \mathrm{~kg}, \mathrm{SD} 2.25$ $\mathrm{kg}$ ). The authors suggest that the type of treatment has a major impact on amount of weight gain 33 . Goodwin et al. 32 looked more closely at the effects of treatment and found that weight gain was associated with positive axillary node status and treatment including prednisone and ovarian ablation. 
A prospective study by Camoriano et al. ${ }^{12}$ evaluated changes in weight during and after a 60-week observation period in premenopausal versus postmenopausal women. The results showed that premenopausal women gained significantly more weight than postmenopausal women did $(5.9 \mathrm{~kg}$ versus $3.6 \mathrm{~kg}$ ). Weight gain appeared to peak at the end of treatment, and while there was a tendency for subsequent weight loss in premenopausal women, measurement after three years of follow up found that the median weight was still $4 \mathrm{~kg}$ above baseline. Premenopausal women who developed amenorrhea gained the same relative amount of weight as those who did not become amenorrheic. This study also included an observational group of postmenopausal women with node positive disease, who did not receive adjuvant treatment. This group gained, on average $1.8 \mathrm{~kg}$, which was less than those receiving adjuvant treatment, but suggested the possibility of factors beyond the influence of chemotherapy that may be a factor in weight gain in women with breast cancer. The etiology suggested by the authors was psychological factors related to surgery and cancer diagnosis.

The impact of psychological factors on weight gain was investigated by Levine et al. 17 who followed 32 women receiving adjuvant chemotherapy, of whom $69 \%$ of gained weight, with a mean weight gain of $1.8 \mathrm{~kg}$. A combination of coping strategies, encompassing emotional discharge, logical analysis, obsessive compulsiveness, and interpersonal sensitivity accounted for $58 \%$ of the variance in overall weight gain. Follow up measures at two years, found that $84 \%$ of the women had gained weight, with a mean weight gain of $6.03 \mathrm{~kg}$. 
Type of chemotherapy, menopausal status, type of surgery, presence of depression, physical activity self report, and self report diet changes did not significantly correlate with weight gain.

The observational accounts of weight gain led to studies that attempted to add more information to the clinical picture. Cheney et al. ${ }^{4}$ were interested in the anthropometric characteristics of this weight gain using computerized tomography scans (CT scans). Regardless, of whether subjects gained or lost weight, there was a tendency to lose lean body mass and gain fat mass during treatment, with increases in abdominal subcutaneous adipose in most participants and slightly larger increases occurring in visceral adipose depots of all the women. Increased fat mass and decreased lean mass, along with a tendency to accumulated fat centrally, are thought to be normal body responses to menopause $1,14,21$. It was concluded that it might be the initial presence of a larger fat mass and larger visceral fat volume that predispose a woman to greater weight gain during breast cancer treatment.

A significant increase in total body water, as measured by deuterium oxide $\left(\mathrm{D}_{2} \mathrm{O}\right)$, and an increase in fat mass, as measured by the sum of skin folds was found by Aslani et al. 45 , who followed 18 women through CMF chemotherapy. A mean weight gain of $2.53 \mathrm{~kg}$ was found with no significant difference noted between pre- and postmenopausal women.

In one of two studies that compared women undergoing chemotherapy to those receiving only localized treatment, Demark-Wahnefried et al. 3 found that over the year of study, premenopausal women gained $2.1 \mathrm{~kg}$ and 
postmenopausal women gained $1.0 \mathrm{~kg}$, but no significant difference was found in measures of resting metabolic rate or dietary intake. However, for those undergoing localized treatment Duel-energy $x$-ray absorptiometry (DXA) results showed that fat mass did not change, and there was a slight decrease in percent body fat, with a slight increase in total and leg lean body mass. Those who had chemotherapy had an increased fat mass and percent body fat, along with decreased leg lean body mass. Investigators proposed that those who had undergone chemotherapy had developed sarcopenic obesity (i.e. weight gain without concurrent gains in lean body mass). All women in the chemotherapy group became amenorrheic, and gradual sarcopenic obesity is noted with increasing age and menopause, however, in this study these normal changes were accelerated as most occurred within 6 months of diagnosis 3,46 . The authors suggested that premature menopause may explain some of the observed changes but also point to the observed decrease in physical activity seen in the chemotherapy group as a significant factor in contributing to a positive energy balance and weight gain, as well as a decrease in lean body mass. From these findings the authors suggest the possible benefits of an intervention to increase physical activity in those undergoing adjuvant chemotherapy treatment for breast cancer ${ }^{3}$. A comparison of activity levels of postmenopausal women supports this view, as women who are physically active have significantly lower percent and total body fat, and higher measures of fatfree mass, than those who are inactive 46 . 
The second study, by Kutynec et al. ${ }^{5}$, found that despite weight maintenance, DXA results showed an increase in total percent body fat and a loss in lean body mass in the leg region in women receiving adjuvant chemotherapy and those receiving radiation. The type of chemotherapy used had a substantially shorter treatment length than that of previous studies, used fewer agents, and was administered intravenously. These are all factors that have been linked to less weight gain during treatment $11,14,33$. No difference in resting energy expenditure, dietary intake, or physical activity was noted between groups.

Research by Goodwin et al. 26 investigated baseline body mass index (BMI), and weight gain during the first year after diagnosis, along with descriptive data on menopausal status, diet, and physical activity measures. Results showed an average weight gain of $1.6 \mathrm{~kg}$ and related changes in BMI, with modest increases in most areas of skin fold thickness measurements. Menopausal status was found to be a significant factor, as those who remained either pre or post menopausal had similar mean gains of $1.07 \mathrm{~kg}$ and $1.05 \mathrm{~kg}$ respectively, whereas those who became menopausal had a mean gain of 2.65 $\mathrm{kg}$. The type of treatment was also of significance, with those receiving any type of chemotherapy gaining the greatest amount of weight, and those receiving only tamoxifen gaining less weight overall, but more than those who received no adjuvant chemotherapy. The investigators looked specifically at caloric intake and physical activity as two potential mechanisms for weight gain. While physical activity did not appear to have an impact, researchers cautioned that 
the self-report technique used to measure physical activity in two 1-week periods might have missed picking up an association. Diet also did not appear to be an important mechanism for weight mediation in those who become menopausal and gained large amounts of weight. It was suggested that further research should concentrate on the possible impact of changes of RMR related to treatment and menopause, which was chosen as a focus for this thesis research.

Other researchers have gone farther in attempts to reveal the possibility of an energy imbalance and its mechanism in relation to weight gain in this population. Foltz 36 identified five variables as potential contributors to weight gain: activity, depression, intake, serum estradiol, and metabolic rate. Of the 34 women studied before and at completion of chemotherapy, $70 \%$ gained weight, with the mean gain in pre- and perimenopausal women of $4.8 \mathrm{~kg}$, compared to a mean gain of $3.6 \mathrm{~kg}$ among postmenopausal women. Changes in depression, activity, RMR and dietary intake were not significantly different between those who gained weight with adjuvant therapy and those who did not. The main finding was that serum estradiol levels were significantly reduced in those women who gained weight, as compared to those who did not. This reduction was greatest among the premenopausal women who gained weight, but it was unclear how this alteration was functionally associated with weight gain. However, Foltz points to the association of estradiol to changes in menopausal status and changes in appetite and dietary intake as possible factors. All 
menstruating women in the study reported less frequent and shorter menses, with an unspecified number experiencing the onset of amenorrhea.

Demark-Wahnefried et al. ${ }^{11}$ sought to answer the question of whether changes in energy intake, physical activity, RMR, and diet-thermogenesis, contribute to the weight gain observed in women receiving adjuvant chemotherapy for breast cancer. Participants did not gain weight during the study, but they also did not lose weight despite a mean reduction in dietary intake. A simultaneous reported decrease in energy expenditure, measured as physical activity, was proposed to be an important factor. It is of note that these women tended to receive shorter and more intense courses of chemotherapy than those previously associated with weight gain. Significant decreases in resting metabolic rate were observed from baseline to mid-treatment, but then returned to levels similar to baseline by the end of the study. There was a trend towards decreased lean body mass, which may be due to the potential effects of chemotherapy on muscle mass, or due to decreased physical activity, and this may account for the initial change in resting metabolic rate. A chart review done at the 1-year mark found weight gain appeared to develop after the study, which researchers concluded may be related to long-term changes in body composition.

Rock et al. 15 investigated weight history, physical activity and dietary intake in women diagnosed with operable breast cancer in the four years previous. Weight gain was reported in $60 \%$ of the subjects, with the overall mean gain of $2.7 \mathrm{~kg}$. The factors that were positively associated with weight 
gain were adjuvant chemotherapy, African-American ethnicity, current energy intake, and postmenopausal status at time of entry into the study. Also a relationship between time since diagnosis and weight gain, suggested that such weight gain is not limited to only during or immediately after initial treatment. The researchers postulated that this pattern was due to the influence of behavioral factors on subsequent energy balance, particularly the observed amount of energy intake and minimal amount of physical activity, both of which are modifiable factors. These investigators also looked at weight history using body mass index (BMI) and patterns of weight change prior to breast cancer treatment. Data from the general population suggests a typical weight gain of 2 $\mathrm{kg}$ over a 10 year period in women between 30 to 55 years 16 . For women in this study, it was found that, on average, there was a $50 \%$ greater weight gain in half the time period. This suggests that after diagnosis and treatment for breast cancer, women tend to gain weight at a rate that is disproportionate to that of women in the general population.

\section{Research Limitations}

To this point, one of the major limitations of the research has been in the collection of dietary and physical activity measures. Most have been based on self-reports. In terms of dietary intake, a reduction in normal intake, or underreporting has been observed as a result of keeping dietary records, and that overweight individuals tend to either underreport or underestimate true intake 47 . Physical activity information has also predominantly been gathered by self reports, which requires accurate recall by the participant, correct 
interpretation of the recorded information by the investigator, and reliance on the equations used to convert this information into energy expenditure or other indicators of physical activity 48 . A valid measure of physical activity that is directly associated with energy output is needed 5,14 .

The measurement of body composition and metabolic rate could also be improved upon. To this point, most studies have looked at resting metabolic rate using less than ideal techniques, often requiring the participant to travel to lab facilities, and tests done without strict control of fasting and recent physical exertion. In terms of body composition, it is only the more recent studies $3,5,11$ that have used Duel-energy $x$-ray absorptiometry (DXA) to determine total and percentage body fat, fat distribution, and fat-free mass, rather than skin fold thickness measures. One study 4 used computerized tomography to investigate changes in abdominal fat accumulation that occurred with weight change.

With other methodological questions aside, the main problem that has faced many of the researchers to date is sample size. It is often difficult to recruit adequate numbers of subjects due to the nature of the disease, and the measures and interventions that are proposed. The reality of the adjuvant treatment is another hurdle for researchers since, for the most part, it is beyond the control of the investigators and can impact significantly on the willingness and ability of subjects to participate in studies.

\section{Summary}

It would appear that most women gain weight with adjuvant chemotherapy treatment for breast cancer. However, the reasons for this are 
unclear, and the phenomenon has been the subject of much research in the past two decades. The major factors that seem to impact on body weight have been identified as, the onset of menopause, and the use of adjuvant chemotherapy. Changes in body composition, and in one case resting metabolic rate, in those undergoing adjuvant therapy have also been noted. Despite these findings, the question of how these factors impact on weight gain has not been satisfactory answered by the literature to date. Is it a factor of changes in energy balance, either increased dietary intake or decreased energy expenditure? If indeed a decrease in energy expenditure is a key factor, does this exert some influence by shifting energy balance, or in the form of decreased lean body mass thereby affecting resting metabolic rate? Or is this weight gain primarily a result of changes in hormonal milieu, which can affect metabolism, appetite and dietary intake? Or is the hormonal shift associated with chemotherapy-related amenorrhea accelerating the normal body composition and weight changes associated with natural menopause? And is resting metabolic rate potentially affected due to the effects of the adjuvant therapy itself or is it due to changes in body composition? These are just some of the many questions that need to be answered. Research to date has established a good base from which further studies can work to add crucial new information. 


\section{Appendix B - TNM Staging for Breast Cancer from the American Cancer}

\section{Society Textbook of Clinical Oncology, $2^{\text {nd }}$ edition 29}

\section{Primary Tumor $(T)$}

TO No evidence of primary tumor

Tis Carcinoma in situ, non-invasive cancer

T1 Tumor $2 \mathrm{~cm}$ of less in greatest dimension

T1a $0.5 \mathrm{~cm}$ or less in greatest dimension

$\mathrm{T} 1 \mathrm{~b}$ More than $0.5 \mathrm{~cm}$, but not more than $1 \mathrm{~cm}$ in greatest dimension

T1c More than $1 \mathrm{~cm}$, but not more than $2 \mathrm{~cm}$ in greatest dimension

T2 Tumor more than $2 \mathrm{~cm}$, but not more than $5 \mathrm{~cm}$ in greatest dimension

T3 Tumor more than $5 \mathrm{~cm}$ in greatest dimension

T4 Tumor or any size with direct extension to chest wall or skin

T4a Extension to chest wall

T4b Edema or ulceration of skin of breast

T4c Both T4a and T4b

T4d Inflammatory carcinoma

\section{Regional Lymph Nodes (N)}

No No regional lymph node metastasis

N1 Metastasis to moveable ipsilateral axillary lymph node(s)

N1a Only micrometastasis (none larger than $0.2 \mathrm{~cm}$ )

N1b Metastasis to lymph nodes(s), any larger than $0.2 \mathrm{~cm}$

N1bi Metastasis in 1 to 3 lymph nodes $(>0.2 \mathrm{~cm}$, but $<2 \mathrm{~cm})$

N1bii Metastasis in 4 or more lymph nodes $(>0.2 \mathrm{~cm}$, but $<2 \mathrm{~cm})$

N1biii Extension of tumor beyond capsule of lymph node metastasis

$\mathrm{N} 1$ biv Metastasis to a lymph node $2 \mathrm{~cm}$ or more in greatest dimension

N2 Metastasis to ipsilateral axillary lymph nodes that are fixed to one another or other structures

N3 Metastasis to ipsilateral internal mammary lymph node(s) 
Distant Metastasis (M)

MO No distant metastasis

M1 Distant metastasis (includes metastasis to ipsilateral supraclavicular lymph nodes(s)

Staging Grouping

$\begin{array}{llll}\text { Stage 0 } & \text { Tis } & \text { N0 } & \text { M0 } \\ \text { Stage I } & \text { T1 } & \text { N0 } & \text { M0 } \\ \text { Stage IIA } & \text { T0 } & \text { N1 } & \text { M0 } \\ & \text { T1 } & \text { N1 } & \text { M0 } \\ & \text { T2 } & \text { N0 } & \text { M0 } \\ \text { Stage IIB } & \text { T2 } & \text { N1 } & \text { M0 } \\ & \text { T3 } & \text { N0 } & \text { M0 } \\ \text { Stage IIIA } & \text { T0 } & \text { N2 } & \text { M0 } \\ & \text { T1 } & \text { N2 } & \text { M0 } \\ & \text { T2 } & \text { N2 } & \text { M0 } \\ & \text { T3 } & \text { N1 } & \text { M0 } \\ & \text { T3 } & \text { N2 } & \text { M0 } \\ \text { Stage IIIB } & \text { T4 } & \text { Any N } & \text { M0 } \\ & \text { Any T } & \text { N3 } & \text { M0 } \\ \text { Stage IV } & \text { Any T } & \text { Any N } & \text { M1 }\end{array}$




\section{Appendix C-DXA Reliability Study}

\section{Methods:}

Subjects: Fourteen healthy adults ( 8 women and 6 men) between the ages of 22 and 54 years were recruited from the University of British Columbia community to undergo total body scans using duel-energy $x$-ray absorptiometry. Informed consent was obtained for each participant and the study was approved by the University of British Columbia Ethics Committee. Participants wore light clothing, free of metal snaps or zippers and removed jewellery during the scan. The total time per participants was approximately one hour, and data collection took place over a 6-week period.

Densitometer: All scans were performed on a single Hologic QDR 4500W densitometer (Hologic Inc., Waltham MA) located in the University of British Columbia bone densitometry laboratory. A dedicated anthropometric step phantom was scanned daily during data collection to satisfy the mandatory daily Hologic quality control procedures and body composition calibrations.

Scan Acquisition: All scans were performed by one of two trained and certified densitometrists. For any one subject, all positioning and scanning was performed by a single technologist. Three total body scans were acquired, in between which participants removed themselves from the table to allow for complete repositioning. All scans were acquired in the array mode. 
Positioning: Positioning protocols from the Hologic QDR 4500 Operator's Manual were followed. Participants were positioned within the black lines outlining the table perimeter, palms of hands facing down, and with at least $6 \mathrm{~cm}$ of space between the top of the head and the positioning line at the top of the table. Feet were angled $30^{\circ}$ and secured with a Velcro strap.

Analysis: All scans were analyzed by one bone densitometry technologist according to Hologic guidelines, using software versions V8.26a:5. Precision was determined by calculating the coefficient of variation expressed as a percentage $(\% \mathrm{CV})$ for the total body lean, fat, and total mass, along with $\%$ fat. The mean and standard deviation of each variable resulting from the three scans was calculated.

$\% \mathrm{CV}=[$ Standard deviation $/$ mean $] \times 100$

The grand mean \% CV was then calculated using all participants' mean $\% \mathrm{CV}$ to represent the overall precision for each variable.

\section{Results:}

Subjects: The eight women participating had a mean height of $164.7 \mathrm{~cm}$ (SD 9.7 , range $=143-172.7)$, a mean weight of $55.4 \mathrm{~kg}(\mathrm{SD} 3.46$, range $=51-61)$ and a mean age of 28.4 years $(S D 7.7$, range $=22-44)$. For the six men the corresponding values were $175.6 \mathrm{~cm}$ (SD 14.1, range $=147-183), 72.3 \mathrm{~kg}$ (SD 10.2 , range $=63-87)$ and 34.7 years $(S D 12.0$, range $=23-54)$. 
In Vivo: The mean percent coefficient of variation (\% CV) and standard deviations for fat mass, lean mass and total mass are presented in Table 1. A \% $\mathrm{CV}$ value for only women participants, and values for six specific body regions are also included.

Table 7 - Percent coefficient of variation (\% CV) and standard deviation (SD) for fat mass, lean mass, total mass and percent body fat.

\begin{tabular}{c|ccccc}
\hline Participant & Area & Fat mass & Lean mass & Total mass & \% fat \\
\hline All & Total Body & $1.56(0.70)$ & $0.36(0.16)$ & $0.14(0.07)$ & $1.56(1.19)$ \\
& L arm & $4.47(1.81)$ & $2.93(1.22)$ & $2.46(1.15)$ & $4.59(2.24)$ \\
& R arm & $9.35(6.71)$ & $2.39(1.05)$ & $2.29(0.91)$ & $7.57(4.10)$ \\
& Trunk & $3.52(1.25)$ & $1.12(0.48)$ & $0.91(0.43)$ & $3.09(1.46)$ \\
& L leg & $3.84(1.72)$ & $2.01(0.73)$ & $1.29(0.52)$ & $3.85(2.05)$ \\
& R leg & $3.22(1.76)$ & $2.13(0.95)$ & $1.47(0.76)$ & $3.53(3.53)$ \\
& Total Body & $1.33(0.48)$ & $0.36(1.20)$ & $.10(0.05)$ & $1.42(1.65)$ \\
& L arm & $3.91(1.31)$ & $2.58(1.47)$ & $1.89(1.30)$ & $4.47(2.21)$ \\
& R arm & $5.87(5.11)$ & $2.43(1.19)$ & $2.40(1.04)$ & $5.82(3.42)$ \\
& Trunk & $3.16(1.54)$ & $0.91(0.53)$ & $0.88(0.41)$ & $2.55(2.06)$ \\
& L leg & $3.03(1.94)$ & $1.99(0.87)$ & $1.21(0.63)$ & $3.32(2.31)$ \\
& R leg & $2.55(0.92)$ & $1.69(0.84)$ & $1.21(0.56)$ & $2.48(1.63)$ \\
\hline
\end{tabular}


Analysis: Using data from all participants, total body measures of fat mass, lean mass and total mass had the lowest variability $(<2 \%)$. Regional analysis showed good agreement for lean and total mass $(<3 \%)$, but reproducibility was less precise for fat mass ( $>3 \%)$. Analysis of women participants only, showed similar results, with good agreement of total body measures of fat, lean and total mass $(<2 \%)$, but greater variability in regional measures, especially fat mass (ranging from $2.55 \%$ in the right leg to $5.87 \%$ in the right arm). The calculation of percent overall body fat was more precise for total body fat $(1.56 \%$ for all participants and $1.42 \%$ for women participants alone) than it was for regional analysis (ranging from $3.09 \%$ in the trunk to $4.59 \%$ in the right arm when all participants were considered and from $2.48 \%$ in the right leg to $5.82 \%$ in the right arm for women).

\section{Conclusion:}

In order to look effectively at changes in body composition over time, it is necessary to establish the precision of the DXA. By performing three scans of the same individual, the variability of the output was assessed. From this the plausibility of true change over time, as measured by repeated scans, can be established. Total body measures of fat mass, lean mass, and total body mass had the lowest variability. Based on the coefficient of variability, the sample size needed to demonstrate a change over time varies. Chilibeck et al. ${ }^{23}$ proposed the sample size needed to show change ranging from 1 to $5 \%$ in total lean and fat mass. Significant to this reliability analysis, a sample size of 11 could demonstrate a $2 \%$ change in total lean mass, and a sample size of 9 could 
demonstrate a $3 \%$ change in total fat mass. However, the change in regional tissues is much harder to prove, as demonstrated by the higher coefficients of variance. And within this the measurement of lean mass has less variance than that of fat mass. Much higher samples sizes are therefore required to demonstrate change in regional areas 23 . In this sample, measures of the right arm had more variability than any other area, and consequently had the highest coefficients of variance. Perhaps the right arm, which is on the far side of the scanner, away from the technologist, is the least likely area to be repositioned correctly. 


\section{Appendix D - Raw Data}

Table 8 - Participant characteristics

\begin{tabular}{c|ccccc}
\hline Participant & Age (years) & $\begin{array}{c}\text { Height } \\
(\mathrm{cm})\end{array}$ & Weight (kg) & $\begin{array}{c}\text { Body Fat } \\
(\%)\end{array}$ & $\begin{array}{c}\text { Menopausal } \\
\text { Status }\end{array}$ \\
\hline 1 & 51 & 157.5 & 101.2 & 46.9 & No \\
2 & 51 & 172.2 & 69.0 & 32.9 & No \\
3 & 50 & 166 & 57.7 & 26.2 & No \\
4 & 54 & 163 & 85.1 & 39.8 & Yes \\
5 & 40 & 176 & 62.9 & 26.6 & No \\
\hline
\end{tabular}

Table 9 - Weight change over the course of treatment $(\mathrm{kg})$

\begin{tabular}{c|ccccccc}
\hline Participant & Pre & $1^{\text {st }}$ & $2^{\text {nd }}$ & $3^{\text {rd }}$ & $4^{\text {th }}$ & $5^{\text {th }}$ & Last \\
\hline 1. & 101.0 & 96.93 & 96.93 & 96.93 & 98.52 & 97.38 & 98.29 \\
2 & 69.01 & 68.78 & 71.05 & 71.50 & 73.77 & 75.59 & 75.82 \\
3 & 57.66 & 57.20 & 56.30 & 57.43 & 58.11 & 58.33 & 58.60 \\
4 & 85.13 & 85.81 & 85.35 & 86.49 & 86.03 & & \\
5 & 62.88 & 63.79 & 64.92 & 66.06 & 66.73 & & \\
\hline
\end{tabular}

Table 10 - Change in resting metabolic rate over the course of treatment (kcal/day)

\begin{tabular}{c|ccccccc}
\hline Participant & Pre & $1^{\text {st }}$ & $2^{\text {nd }}$ & $3^{\text {rd }}$ & $4^{\text {th }}$ & $5^{\text {th }}$ & Last \\
\hline 1 & 1423.51 & 1316.57 & 1514.25 & 1140.42 & 1417.43 & NA & 1341.77 \\
2 & 1325.75 & 1497.35 & 1222.69 & 1317.04 & 1288.18 & 1119.43 & 1244.51 \\
3 & 1038.15 & 1183.06 & 1158.01 & 1257.12 & 978.74 & 972.87 & 1409.64 \\
4 & 1379.41 & 1535.08 & 1627.51 & 1216.28 & 1201.63 & & \\
5 & 1193.63 & 897.6 & 1277.56 & 1199.88 & NA & & \\
\hline
\end{tabular}

